\title{
Analog VLSI Models of Range-Tuned Neurons in the Bat Echolocation System
}

\author{
Matthew Cheely \\ Neurosciences and Cognitive Sciences Program, University of Maryland, College Park, MD 20742, USA \\ Email: mcheely@isr.umd.edu \\ Timothy Horiuchi \\ Department of Electrical and Computer Engineering, University of Maryland, College Park, MD 20742, USA \\ Institute for Systems Research, University of Maryland, College Park, MD 20742, USA \\ Email: timmer@isr.umd.edu
}

Received 3 May 2002 and in revised form 20 February 2003

\begin{abstract}
Bat echolocation is a fascinating topic of research for both neuroscientists and engineers, due to the complex and extremely timeconstrained nature of the problem and its potential for application to engineered systems. In the bat's brainstem and midbrain exist neural circuits that are sensitive to the specific difference in time between the outgoing sonar vocalization and the returning echo. While some of the details of the neural mechanisms are known to be species-specific, a basic model of reafference-triggered, postinhibitory rebound timing is reasonably well supported by available data. We have designed low-power, analog VLSI circuits to mimic this mechanism and have demonstrated range-dependent outputs for use in a real-time sonar system. These circuits are being used to implement range-dependent vocalization amplitude, vocalization rate, and closest target isolation.
\end{abstract}

Keywords and phrases: neuromorphic engineering, echolocation, bat, biosonar, delay tuning.

\section{INTRODUCTION}

Information about target range has many uses for bats during both prey capture and navigation tasks. Beyond the extraction of distance and velocity, it may be important for less obvious tasks, such as optimizing the parameters of the echolocation process. For example, as a bat approaches a target, it alters the repetition rate, duration, spectral content, and amplitude of its vocalizations [1]. Echolocation is not only used for insect capture but also provides information to the bat about obstacles, roosts, altitude, and other flying creatures.

Neurons have been found in bats that show a "facilitated" response to paired sounds (a simulated vocalization and an echo) presented at particular delays. The cells' responses to sounds presented at the appropriate delays are much greater than the sum of responses to the individual sounds presented alone. These cells are part of a larger class of neurons called "combination-sensitive" neurons, and are specifically referred to as delay-tuned cells. Delay-tuned cells are found at many levels in the bat auditory system. They have been found in the inferior colliculus (IC) [2], the medial geniculate body (MGB) [3], and the auditory cortex [4]. Disruption of cortical delay-tuned cells has been shown to impair a bat's ability to discriminate artificial pulse-echo pair delays [5]. It is likely that delay-tuned neurons play a role in forming the bat's perception of range although delay-tuned cells have also been shown to respond to the social calls of other bats $[6,7]$.

The largest amount of information related to mechanisms underlying the delay-tuned response comes from the mustached bat (Pteronotus parnellii) $[2,8,9,10,11,12,13$, $14]$. In this species, delay-tuned cells respond specifically to the first harmonic of the echolocation call $\left(\mathrm{FM}_{1}\right)$ followed by a delayed higher harmonic $\left(\mathrm{FM}_{2-4}\right)$ [15]. In contrast, delaytuned neurons in the big brown bat (Eptesicus fuscus) respond preferentially to an initial loud sound and a delayed softer sound, with no clear frequency relationship [16]. Both of these mechanisms are thought to relate to discrimination between outgoing calls and returning echoes. In the case of the mustached bat, the $\mathrm{FM}_{1}$ component of the outgoing call is weak, and could be attenuated enough in the returning echo not to impact the ranging system. Obviously, in the case of Eptesicus fuscus, the outgoing call will be much louder than returning echoes.

In at least one study of the central nucleus of the IC of the mustached bat, the majority $(76 \%)$ of cells were found to be combination-sensitive, and approximately $46 \%$ of those were delay-tuned [12]. The responses of these delay-tuned cells have been well characterized $[2,10,12]$. In response to an $\mathrm{FM}_{1}$ tone, they have no response, or respond weakly at latencies as long as 30 milliseconds [12]. In response to tones 


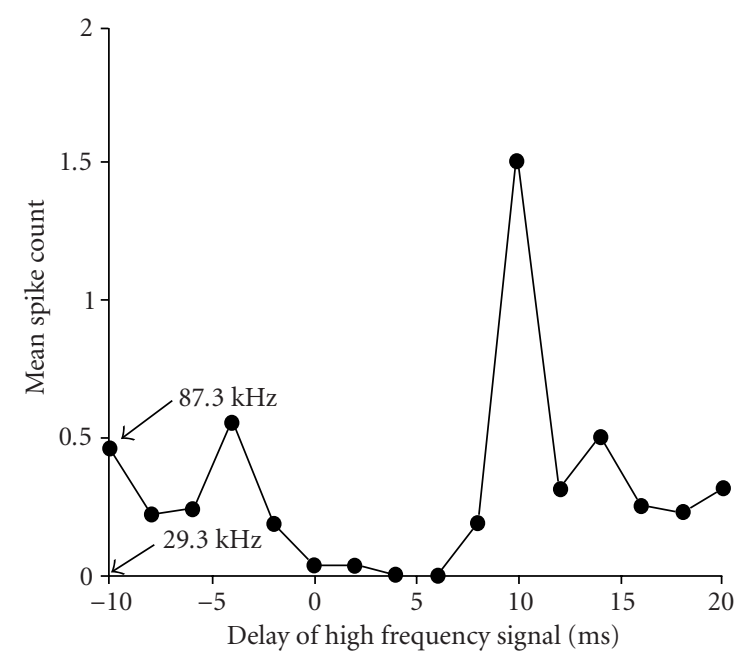

Figure 1: From Portfors and Wenstrup [12]. Tuning curve of a delay-tuned neuron in mustached bat IC. Average number of spikes is plotted versus the time interval between the $\mathrm{FM}_{1}(29.3 \mathrm{kHz})$ and $\mathrm{FM}_{n}(87.3 \mathrm{kHz})$ presentations. Arrows indicate the average number of spikes in response to each stimulus presented alone.

from higher harmonics of the echolocation calls $\left(\mathrm{FM}_{2-4}\right)$, the cells respond weakly with consistent short latencies averaging about 7 milliseconds [12]. The latency of response to the $\mathrm{FM}_{1}$ tone is highly correlated with the pulse-echo delay to which the neuron best responds (best delay or $\mathrm{BD}$ ), while the latency of response to the $\mathrm{FM}_{n}$ component stays relatively constant across neurons, both in the IC and MGB $[3,12]$. It has been proposed that the neuron responds best when this long-latency response to the $\mathrm{FM}_{1}$ component and the short-latency response to the higher harmonic coincide in time. This allows one to predict the $\mathrm{BD}$ of the neuron as latency $\mathrm{FM}_{1}$-latency $\mathrm{FM}_{n}$. The biological data supports this "latency-coincidence" hypothesis $[3,12]$. It is unusual that there are such long-latency responses in IC, considering that the latencies of most neurons in brainstem regions that project to IC are on the order of 3-7 milliseconds [17, 18]. It is unlikely that the mono and disynaptic pathways from cochlear nucleus to IC could create the long latencies seen in IC.

Figure 1 is a tuning curve from a delay-tuned neuron showing the common property of suppression following presentation of the $\mathrm{FM}_{1}$ component and prior to the facilitative effect $[3,12]$. During the period of suppression following the $\mathrm{FM}_{1}$ signal, the cell's response to an $\mathrm{FM}_{n}$ signal decreases. This suppression is consistent with a period of inhibition that is responsible for the creation of the long latency response to the $\mathrm{FM}_{1}$ component of the outgoing call. One proposed model for generating long latencies using inhibition is the postinhibitory-rebound (PIR) model [19]. In this model, the outgoing vocalization triggers an inhibitory period in delaytuned neurons. An instability in the membrane dynamics of the delay-tuned neuron leads to a brief depolarizing jump above resting potential following the release of inhibition. This type of rebounding behavior has been documented or

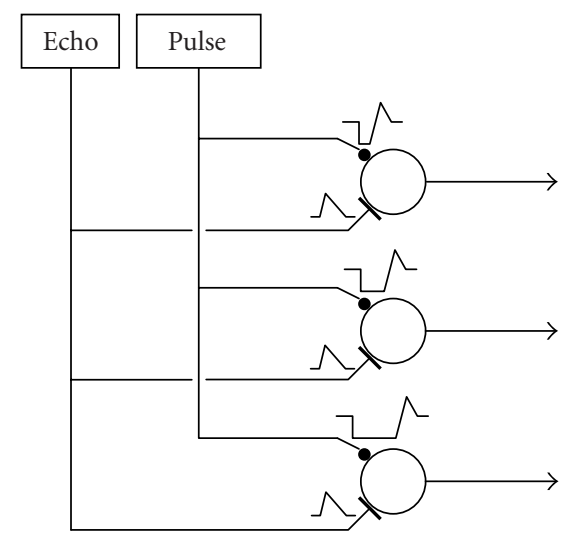

FIGURE 2: Schematic of the PIR network model for delay-tuned cells. Delay tuning is created by vocalization-triggered inhibitory connections of varying duration which project to the delay-tuned cells. Rebound activity at the end of inhibition creates a facilitation window during which excitatory input from returning echoes can arrive and trigger a spike. The $\mathrm{BD}$ of the cell is the difference in the rebound and excitatory input latencies.

inferred in many types of neurons. In the model, if an excitatory input from a returning echo coincides with the rebound event, the membrane potential will cross threshold, and the cell will fire a spike. The delay to which the neuron is tuned is determined by the duration of the inhibitory input that it receives. Figure 2 presents a schematic of the PIR model for delay tuning.

The PIR model for delay tuning predicts that blocking of inhibition should reduce or eliminate the latency-coincident facilitation in the neuron. Results from a study by Wenstrup and Leroy [14] indicate that blocking the inhibitory transmitter glycine through application of strychnine has such an effect. Since IC does not contain glycinergic cells [20], the glycinergic inhibition is believed to project from lower centers. Retrograde studies of inputs to delay-tuned cells in IC show projections originating in VNLL, INLL, and other lower auditory areas [11]. Studies of VNLLc (a subregion of VNLL known for short and remarkably precise latencies with broad frequency tuning) have shown that it contains glycinergic cells that project broadly and densely to the IC [21].

\section{VLSI CIRCUIT IMPLEMENTATION}

The design of our delay-tuned neuron is intended to capture the fundamental functional aspects of biological delay-tuned neurons while maintaining simplicity of use and maximum control over the neuron's behavior. The analog processing components in the neuron are implemented using transistors operating in the subthreshold regime such that the drain current-voltage relationship is given by

$$
\begin{array}{ll}
I_{d n}=I_{0} e^{\kappa_{n} V_{G B} / V_{T}}\left(e^{-V_{S B} / V_{T}}-e^{-V_{D B} / V_{T}}\right), & \text { for the nFET, } \\
I_{d p}=I_{0} e^{\kappa_{p} V_{B G} / V_{T}}\left(e^{-V_{B S} / V_{T}}-e^{-V_{B D} / V_{T}}\right), & \text { for the pFET. }
\end{array}
$$




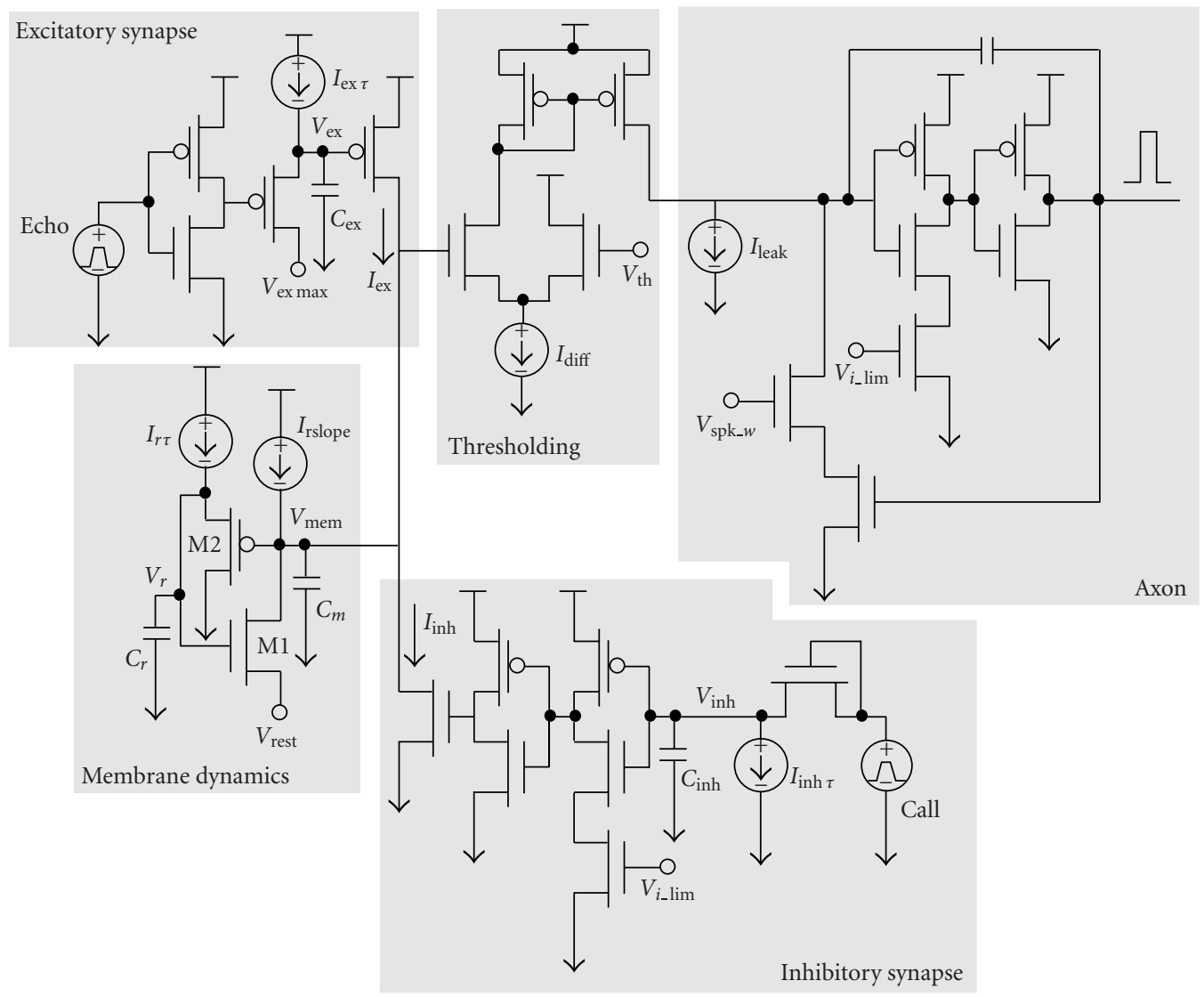

Figure 3: Circuit schematic for the rebound neuron. Circuit was fabricated in an AMI 1.5- $\mu$ m process by MOSIS. An individual neuron measures $71 \times 452 \lambda(53.25 \times 339 \mu \mathrm{m}) .20 \%$ of the circuit size is from capacitors, which can be greatly reduced in later versions of the chip $\left(C_{m}=C_{r}=600 \mathrm{fF}, C_{\text {ex }}=400 \mathrm{fF}, C_{\text {inh }}=440 \mathrm{fF}\right)$.

To simplify analysis, we generally assume that the transistors are operating in saturation and that the drain current can be computed as

$$
\begin{aligned}
& I_{d n}=I_{0} e^{\left(\kappa_{n} V_{G B}-V_{S B}\right) / V_{T}}, \quad \text { for the nFET, } \\
& I_{d p}=I_{0} e^{\left(\kappa_{p} V_{B G}-V_{B S}\right) / V_{T}}, \quad \text { for the pFET. }
\end{aligned}
$$

The delay-tuned neuron circuit is constructed from component circuits designed to mimic the basic functional elements of a PIR delay-tuned neuron. The neuron is shown in Figure 3, with the individual components highlighted. A strong, long duration synapse sets up the basic delay at which the cell will respond. A rebound circuit implements dynamics that generate a facilitation window at the end of inhibition. The excitatory synapse produces a decaying current triggered by returning echoes. A differential pair circuit compares the membrane potential to a threshold voltage, and an axon circuit [22] generates spikes when the membrane potential crosses the threshold.

\section{Inhibitory synapse}

A spike (labeled "call") triggers the inhibitory synapse and drives current through a diode-connected nFET, quickly charging $C_{\text {inh }}$ and driving $V_{\text {inh }}$ to a high voltage $V_{\text {inh } H}$. This signal is passed through two inverters in series, activating an nFET that draws current from the membrane dynamics circuit, pulling $V_{\text {mem }}$ to ground (see Figure 4 for example traces of $V_{\text {mem }}$ ). The bias current $I_{\text {inh }} \tau$ gradually discharges $C_{\text {inh }}$. When $V_{\text {inh }}$ drops below the switching voltage of the inverters, the output nFET is turned off, and the neuron is released from inhibition. Thus, the length of inhibition $t_{\text {inh }}$ is given by approximately

$$
t_{\mathrm{inh}}=\frac{C_{\mathrm{inh}}\left(V_{\mathrm{inh} H}-V_{\mathrm{hl}}\right)}{I_{\mathrm{inh} \tau}}
$$

where $V_{\mathrm{hl}}$ is the inverter's high-to-low transition voltage. This $V_{\mathrm{hl}}$ will depend on the bias voltage $V_{i \_l i m}$, which is used to limit the maximum current in the inverters driven by ana$\log$ inputs. The duration of inhibition is adjusted via $I_{\operatorname{inh} \tau}$.

\section{Membrane dynamics}

The membrane dynamics circuit generates PIR in the cell, opening a facilitation window that leads to delay tuning. In the absence of external input from the excitatory or inhibitory synapses, we can derive the equilibrium state of the membrane circuit. Applying the drain current equation to 


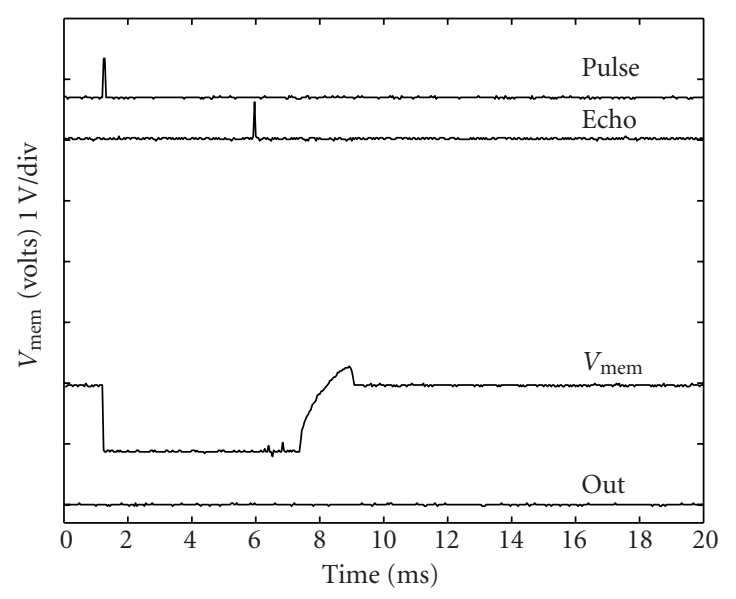

(a)

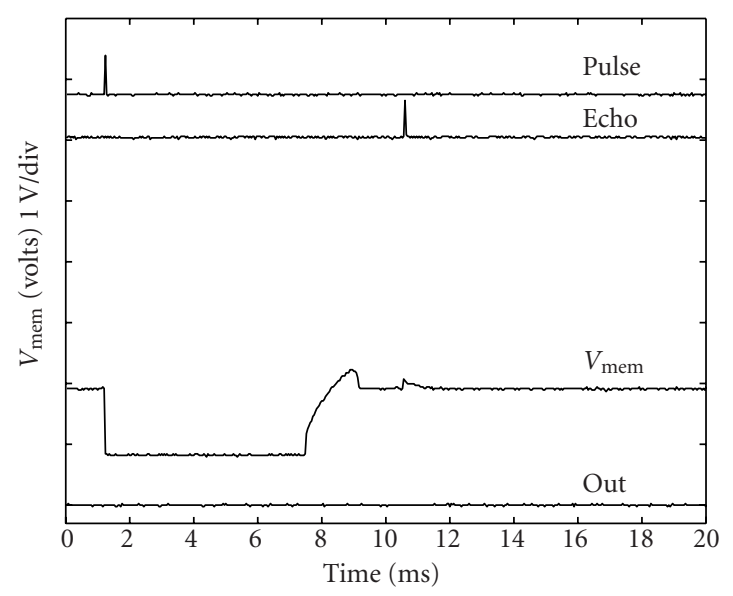

(b)

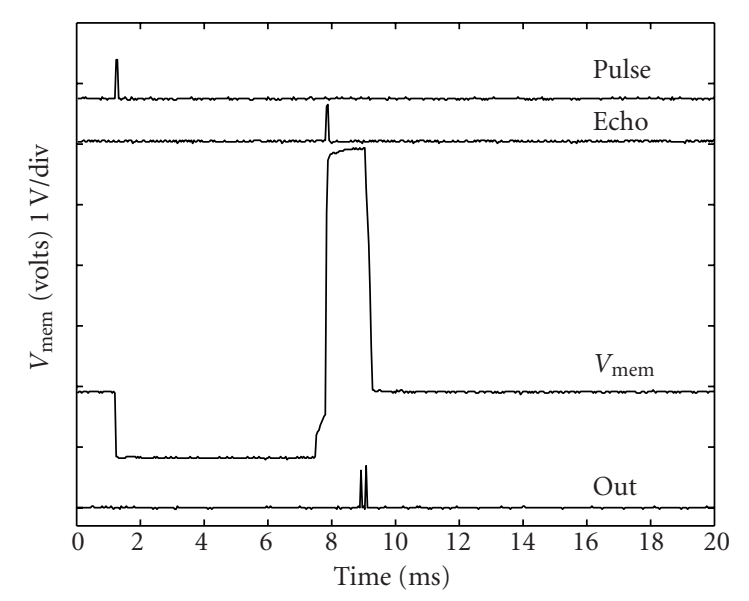

(c)

FIgURE 4: Response of a silicon delay-tuned neuron to artificial stimuli at different delays. Top trace is the spike representing an outgoing pulse. Second trace is a spike representing an echo. Third trace is $V_{\text {mem }}$ of the neuron. Bottom trace shows any output spikes from the neuron. The pulse, echo, and out traces are $0-5 \mathrm{~V}$ digital signals. (a) Stimulus delay is shorter than facilitative delays. (b) Stimulus delay is longer than facilitative delays. (c) Stimulus delay is facilitative. transistor M1 and solving for $V_{r}$, we obtain the equation

$$
V_{r_{\text {_eq }}}=\frac{1}{\kappa_{n}}\left(V_{\text {rest }}+V_{T} \ln \left(\frac{I_{\text {rslope }}}{I_{0}}\right)\right) \text {. }
$$

Following the same process for $\mathrm{M} 2$ and solving for $V_{\text {mem }}$, we obtain

$$
V_{\text {mem }- \text { eq }}=\frac{1}{\kappa_{p}}\left(V_{r_{-} \text {eq }}-V_{T} \ln \left(\frac{I_{r \tau}}{I_{0}}\right)\right)+V_{\text {dd }}\left(1-\frac{1}{\kappa_{p}}\right) .
$$

Substituting (4) into (5), we see that

$$
\begin{aligned}
V_{\text {mem_eq }}= & \frac{1}{\kappa_{n} \kappa_{p}} V_{\text {rest }}+\frac{1}{\kappa_{n} \kappa_{p}} V_{T} \ln \left(\frac{I_{\text {rslope }}}{I_{0}}\right) \\
& -\frac{1}{\kappa_{p}} V_{T} \ln \left(\frac{I_{r \tau}}{I_{0}}\right)+V_{\text {dd }}\left(1-\frac{1}{\kappa_{p}}\right) .
\end{aligned}
$$

Assuming the ideal case where $\kappa_{n}=\kappa_{p}=1$,

$$
V_{\text {mem_eq }}=V_{\text {rest }}+V_{T} \ln \left(\frac{I_{\text {rslope }}}{I_{r \tau}}\right) \text {. }
$$

In the case where $I_{\text {rslope }} \leq I_{r \tau}$, transistor M1 will leave saturation and $V_{\text {mem }}$ will sit slightly above $V_{\text {rest }}$.

The dynamic behavior of the circuit is best described in a stepwise manner. When an outgoing call triggers the inhibitory synapse, $V_{\text {mem }}$ is pulled to ground and $V_{r}$, which is connected to $V_{\text {mem }}$ through a source follower, will also drop to some minimum voltage level $V_{r_{-} \text {min }}$, which depends on $I_{r \tau}$ and is described by the equation

$$
V_{r_{-} \min }=V_{t} \ln \left(\frac{I_{r \tau}}{I_{0}}\right)+V_{\mathrm{dd}}\left(1-\kappa_{p}\right) .
$$

On the release of inhibition, $V_{\text {mem }}$ is less than $V_{\text {rest }}$, and the source and drain of M1 are reversed from the equilibrium state. Current flowing through M1 combined with $I_{\text {rslope }}$ acts to drive $V_{\text {mem }}$ toward $V_{\text {rest }}$ at a rate of

$$
\dot{V}_{\text {mem }}=\frac{I_{\text {rslope }}+I_{0} e^{\kappa_{n} V_{r} / V_{T}}\left(e^{-V_{\text {mem }} / V_{t}}-e^{-V_{\text {rest }} / V_{t}}\right)}{C_{m}} .
$$

It is reasonable to assume that with the appropriate biasing, $V_{\text {mem }}$ will rise faster than $V_{r}$ such that, during the rebound, the current through M2 will be negligible and $V_{r}$ will rise at a rate of

$$
\dot{V}_{r}=\frac{I_{r \tau}}{C_{r}}
$$

Under these conditions, once $V_{\text {mem }}$ reaches $V_{\text {rest }}$, the current through M1 will be negligible and $V_{\text {mem }}$ will rise at a rate of

$$
\dot{V}_{\text {mem }}=\frac{I_{\text {rslope }}}{C_{m}},
$$

where $V_{\text {mem }}$ will continue to rise at a linear rate, exceeding its equilibrium value, until $V_{r}$ reaches $V_{\text {rest }}$ and transistor M1 begins to turn on. The rebound will peak $\left(\dot{V}_{\text {mem }}=0\right)$ when the drain current in M1 equals $I_{\text {rslope, which occurs at }}$ 
the equilibrium voltage for $V_{r}$, given in (4). We define the duration of the rebound $t_{\text {reb }}$ as the time interval after inhibition during which $V_{\text {mem }}$ is rising. This is easily computed provided that $V_{\text {mem }}$ rises faster than $V_{r}$ and the assumption that $V_{r}$ rises linearly holds. The duration of the rebound is then

$$
\begin{aligned}
t_{\text {reb }} & =\frac{C_{r}\left(V_{r_{-} \text {eq }}-V_{r_{-} \text {min }}\right)}{I_{r \tau}} \\
& =\frac{C_{r}\left(V_{\text {rest }}+V_{T} \ln \left(I_{\text {rslope }} / I_{r \tau}\right)+V_{\text {dd }}\left(\kappa_{p}-1\right)\right)}{\kappa_{n} I_{r \tau}} .
\end{aligned}
$$

An estimate for peak voltage of the rebound $V_{m_{\text {_peak }}}$ can be obtained by assuming that $V_{\text {mem }}$ rises to $V_{\text {rest }}$ nearly instantaneously, then applying the value of $\dot{V}_{\text {mem }}$ for $V_{\text {mem }}>V_{\text {rest }}$ given in (11) and $t_{\text {reb: }}$ :

$$
V_{m_{-} \text {peak }}=V_{\text {rest }}+\frac{I_{\text {rslope }}}{C_{m}} t_{\text {reb }} .
$$

The parameters of the circuit are adjusted so that $V_{m \text {-peak }}$ is less than the threshold voltage.

The neuron will be most responsive to an excitatory current during the rising portion of the rebound. Normally, the active membrane properties dampen the response of the cell to excitatory currents. However during the rebound period, the active processes of the membrane have not recovered sufficiently to compensate for excitatory input. During this time window, $I_{\text {ex }}$ sums with $I_{\text {rslope }}$ to drive $V_{\text {mem. }}$. When $V_{\text {mem }}>V_{\text {rest }}, \dot{V}_{\text {mem }}$ can be computed as

$$
\dot{V}_{\text {mem }}=\frac{I_{\text {ex }}+I_{\text {rlope }}}{C_{m}} .
$$

In this condition, the current through M1 must compensate for $I_{\text {ex }}+I_{\text {rslope }}$ before the membrane voltage peaks. If we assume a constant excitatory current, the rising time of the system, $t_{r}$, would be

$$
t_{r}=\frac{C_{r}\left(V_{\text {rest }}+V_{T} \ln \left(\left(I_{\text {ex }}+I_{\text {rslope }}\right) / I_{r \tau}\right)+V_{\text {dd }}\left(\kappa_{p}-1\right)\right)}{\kappa_{n} I_{r \tau}} .
$$

As in (13), we can form an estimate of the peak voltage that the membrane reaches:

$$
V_{m_{-} \text {peak }}=V_{\text {rest }}+\frac{I_{\text {ex }}+I_{\text {rslope }}}{C_{m}} t_{r} .
$$

We can see that $V_{\text {mem }}$ now rises at a faster rate for a longer period of time than in (13). Under these conditions, $V_{\text {mem }}$ easily exceeds threshold before $V_{r}$ increases enough to compensate. Regardless of the actual time course of $I_{\mathrm{ex}}$, the response of the neuron to excitatory inputs during this time window is facilitated over the normal condition.

Once the rising portion of the rebound ends, $V_{\text {mem }}$ begins to fall and $V_{r}$ continues to rise until $I_{r \tau}$ is balanced by the current in M2. Once this point is reached, $V_{r}$ begins to fall, following $V_{m}$ quickly. Both voltages return to their equilibrium value with no observable ringing. This is because

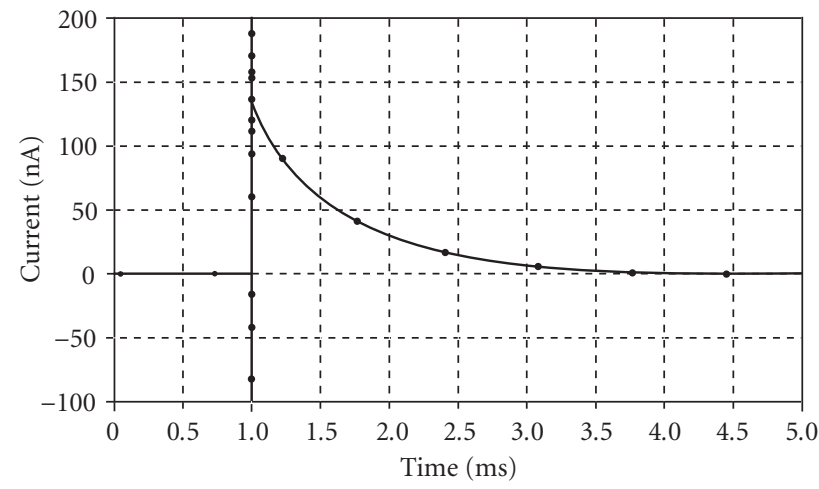

FIGURE 5: Simulated excitatory postsynaptic current (EPSC) triggered by a single spike. The current decays exponentially with a controllable time constant. The transients at 1 millisecond are due to capacitive coupling of the input signal, and extend from $-0.2 \mu \mathrm{A}$ to $0.8 \mu \mathrm{A}$.

the source follower acts rapidly in the downward direction, and due to the fact that M1 cannot drive $V_{\text {mem }}$ below $V_{\text {rest }}$, which is generally close to the equilibrium state, making the amount of overshoot necessary for ringing essentially impossible to achieve.

\section{Excitatory synapse}

In the excitatory synapse, an incoming spike (labeled "echo") is inverted to activate a pFET and drive $V_{\mathrm{ex}}$ from $V_{\mathrm{dd}}$ to $V_{\text {ex max }}$. After the spike, the bias current $I_{\text {ex } \tau}$ charges $V_{\text {ex }}$ linearly back to $V_{\mathrm{dd}}$. This linearly rising voltage gives rise to an exponentially decaying current in the output pFET:

$$
\begin{gathered}
I_{\mathrm{ex}}(t)=I_{0} e^{\kappa_{p}\left(V_{\mathrm{dd}} / V_{T}-\left(V_{\mathrm{ex} \max } / V_{T}+\left(I_{\mathrm{ex} \tau} / V_{T} C_{\mathrm{ex}}\right) t\right)\right)}=I_{\max } e^{\left(-\kappa_{p} I_{\mathrm{ex} \tau} / C_{\mathrm{ex}}\right) t} \\
I_{\max }=I_{0} e^{\kappa_{p}\left(V_{\mathrm{dd}}-V_{\mathrm{ex} \max }\right) / V_{T}} .
\end{gathered}
$$

This decaying current drives $V_{\text {mem }}$ in the membrane dynamics circuit. A simulation of the excitatory postsynaptic current (EPSC) is shown in Figure 5.

\section{Biasing and power consumption}

Based on the above analysis, we can see that the shape of the tuning curve for the neuron depends on several factors. The duration of inhibition sets the time when the rebound period starts, and is used to adjust the "BDs" of the neurons. The width of the tuning curve depends on the durations and magnitudes of the EPSC and the rebound period. For a fixed rebound, the shape of the EPSC determines the length of time prior to the end of inhibition during which an echo can elicit a spike. For a neuron with a longer EPSC, echoes can arrive earlier, and still integrate enough charge onto $C_{m}$ to drive the membrane voltage past threshold before the rebound ends. For an echo arriving after the release of inhibition, the amount of overlap between the EPSC and the rebound is determined by the duration of the rebound. This, in turn determines how long after the release of inhibition an echo can arrive and still elicit a spike. 


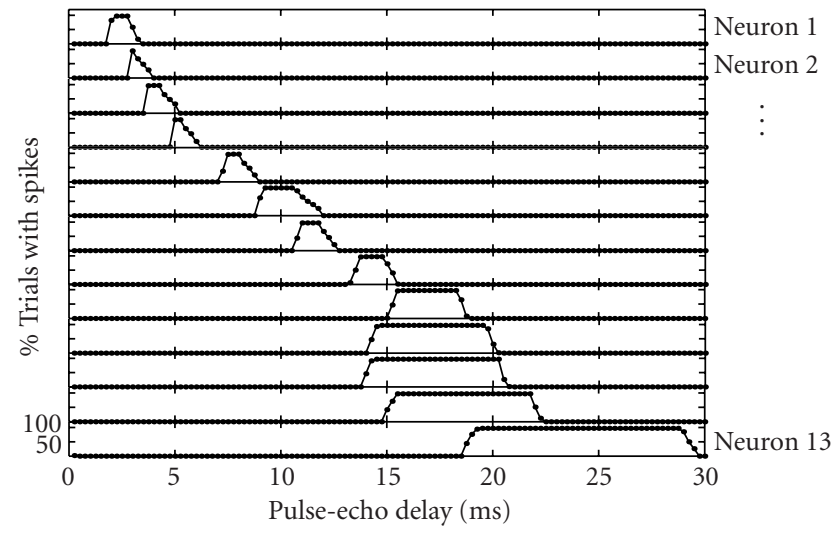

FIgURE 6: Example tuning curves for the 13-neuron array. Each neuron was presented with 100 pulse-echo spike pairs at delays ranging from 0 to 30 milliseconds in 0.25 millisecond intervals. Percent of trials in which the neuron spiked are plotted versus time. (The peak in each plot reaches $100 \%$.)

Most current biases in the chip are the same in each neuron and are created with $n$-channel and $p$-channel transistors whose gate voltages are set off chip. The currents $I_{\text {inh } \tau}$ and $I_{\mathrm{ex} \tau}$ change across the neuron array, and are biased using a cascaded series of current mirrors with a further small bias current added in at each stage. This creates an approximately linearly increasing set of currents for $I_{\operatorname{inh} \tau}$, which tunes the array of neurons to different delays, and for $I_{\mathrm{ex} \tau}$, which sets wider tuning curves at longer delays while preventing long EPSCs at short delays that could trigger a short-delay neuron based on the outgoing call.

The entire neuron array with biasing circuitry measures $927 \times 389.25 \mu \mathrm{m}$ in the AMI $1.5-\mu \mathrm{m}$ process. A large portion of the layout size is due to capacitors which can be significantly reduced in later designs. Simulations indicate that quiescent power consumption in the circuit is on the order of $33 \mu \mathrm{W}$. At an echolocation rate of $50 \mathrm{~Hz}$ with a single target echo, simulated power consumption is about $550 \mu \mathrm{W}$.

\section{CHIP PERFORMANCE}

In order to test chip performance and aid in biasing, the membrane voltages, $V_{\text {mem }}$ of the neurons were passed offchip with amplifiers activated by a scanner mechanism that allows sequential examination of the array elements. Oscilloscope traces of an example neuron's membrane voltage, along with its pulse, echo, and output spikes, are shown for several delays in Figure 4.

Sample tuning curves for the 13-neuron array are shown in Figure 6. Long-delay neurons have wider tuning curves than short-delay neurons. Tuning curves are plotted as the percent of trials eliciting spikes versus pulse-echo delay for more relevant comparison to biological delay-tuned cells, which usually respond with either one or no spikes on each stimulus presentation. The rebound chip can be tuned to respond with one or multiple spikes to a facilitative stimulus.

\section{THE NARROWBAND ECHOLOCATION SYSTEM}

We have incorporated this chip into a sonar system to provide the necessary input signals and to convert the outputs into appropriate signals for reporting range and for modifying the sonar parameters. In this section, we describe the external components that the chip interacts with to produce an estimate of range and describe how this information is used to drive the sonar system. A block diagram of the components in the system is shown in Figure 7.

The narrowband sonar system we are using is part of a larger echolocation project that is developing additional brainstem and midbrain neural models for the bat echolocation system, such as the binaural azimuthal localization circuitry of the lateral superior olive (LSO) (Horiuchi and Hynna [23]). In our system, the sound received by the microphones is converted into an envelope signal which is passed to high-threshold and onset-type neurons. Figure 8 shows traces of the vocalization signal from the system, the output of the envelope board, and the resulting high-threshold and onset neuron spikes.

\section{Triggering}

The pulse input to the delay-tuned chip that begins the timing in the system is based on the high-intensity signal, received by the microphones following the vocalization, and not on the electrical signal that triggers the vocalization. There are several reasons for this arrangement: (1) most of the data on delay-tuned cells has been obtained in an experimental paradigm where the bat does not emit vocalizations and both the pulse and echo sounds are generated by the experimenter, (2) variations in the latency from trigger signal to the actual sound generation from the larynx of a real bat could significantly affect the accurate perception of range ( 1 millisecond delay $=\sim 16 \mathrm{~cm}$ in range). Basing the delay measurement on the actual time of emission is probably more accurate and also provides a natural mechanism for compensating for changes in the FM sweep rate.

\section{Echoes}

The echo signal input to the chip comes from an onset neuron that responds with a single spike at the beginning of a sound. The onset response is triggered by a positive derivative on a signal of sufficient magnitude. The onset neuron also responds to the vocalization signal, but the simultaneous inhibition triggered by the high-threshold neuron masks this input, provided that the EPSC is shorter than the inhibitory period. The use of a single-spike response in the onset neuron and in the high-threshold neuron allows the system to transmit only timing information to the delaytuned chip, discarding possibly confounding information related to the duration of the signal or its amplitude.

\section{First-target isolation}

In order to effectively control vocalization parameters in the ranging system, external circuitry is used to restrict the chip's 

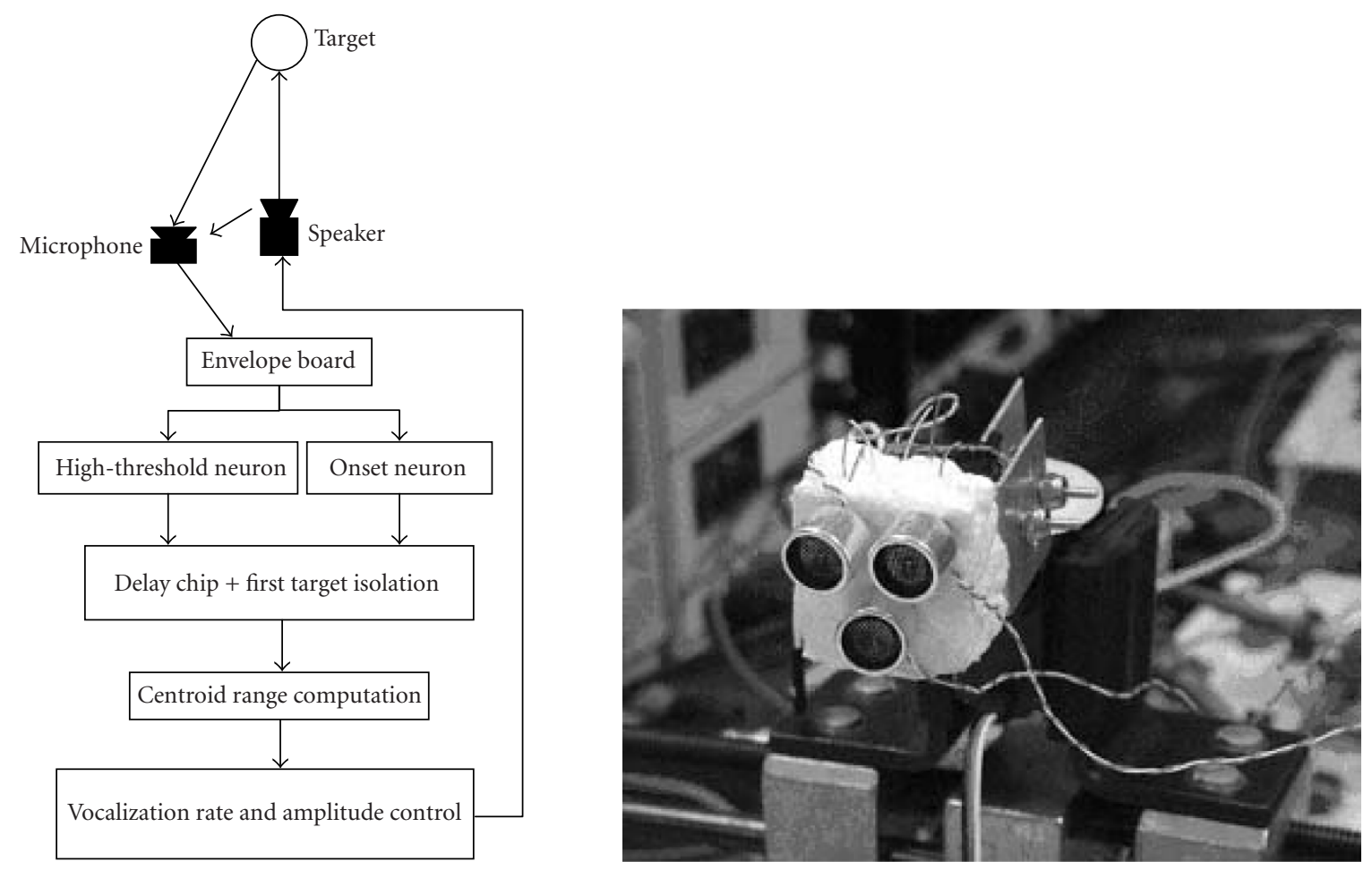

FIGURE 7: Block diagram of ranging system components, and photograph of sonar head. The top two components on the sonar head are ultrasonic microphones. The lower single component is the speaker. The head is mounted on a servo "neck" mechanism used for target tracking.

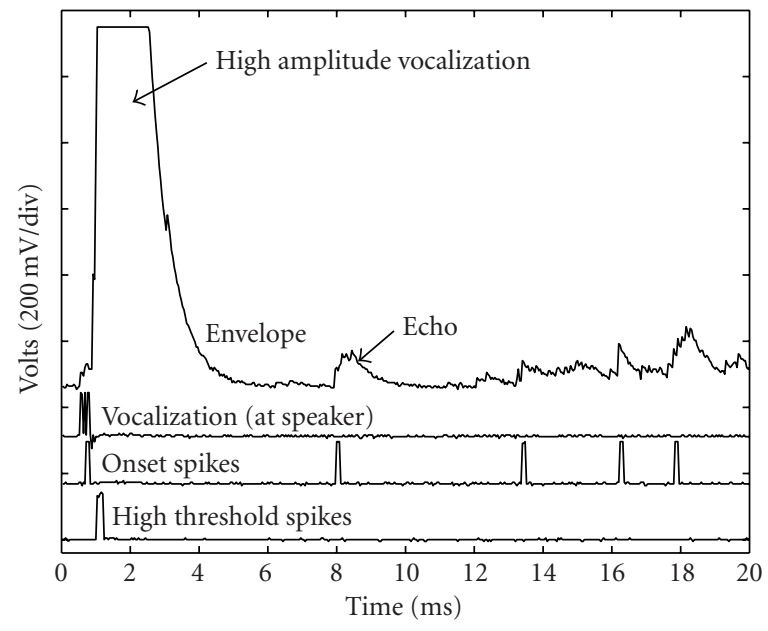

FIgURE 8: Output of envelope board and corresponding spikes. The top trace is the output of the envelope board in response to the sounds following a vocalization. The second trace is the signal that drives the $40-\mathrm{kHz}$ ultrasonic speaker. The third trace shows spikes from the onset neuron in response to echoes. The bottom trace shows spikes from the high-threshold neuron. Note that it responds only to the initial high-intensity sound from the outgoing vocalization. The vocalization, onset, and high-threshold spikes are $0-5 \mathrm{~V}$ digital signals.

input to the nearest target (i.e., first echo) and allows only the information from that signal to impact the delay-tuned circuit. Since the delay-tuned cells respond at approximately the same time as the delay to which they are tuned, the first delay-tuned cell that spikes should be in response to the closest target. Once a delay-tuned cell spikes, the excitatory synapses for the whole array are deactivated by switching $V_{\text {ex max }}$ to $V_{\text {dd }}$. This prevents any further inputs from having an effect, but allows active synapses to finish out their time course of operation. On the next pulse signal, the synaptic block is removed. This process ensures that any spikes that occur in the delay-tuned chip are in response only to the first detected echo.

This isolation circuit acts as a type of attentional filter only allowing a subset of the available sensory data through. In this implementation, the attentional window is timecontrolled, however spatially-mediated attentional mechanisms are also easily implemented, and would be more naturally implemented in a neural system, particularly for attending to objects at intermediate ranges between two clutter targets.

\section{Range centroid}

Because the tuning curves of some delay-tuned neurons overlap, a single echo can trigger a spike from multiple neurons on the ranging chip. In order to combine the information from multiple spikes into a single estimate of range, a resistive network with transmission gates and a leak to ground is used to compute a "centroid" range estimate. Spikes from 


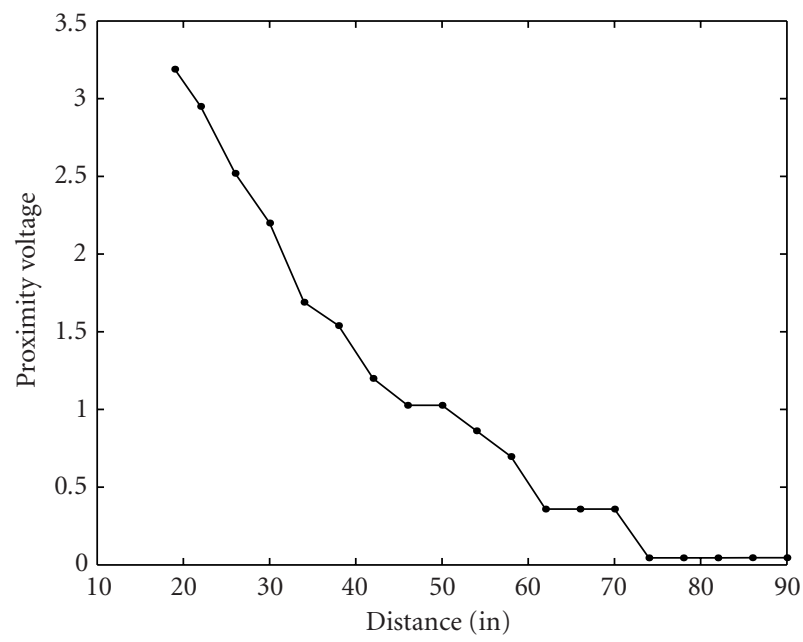

FIGURE 9: Voltage output of the centroid range estimate plotted against target distance. The centroid board has been biased with a linear array of voltages, which assumes a linear set of BDs for the neurons. This estimate works well for closer ranges, but loses accuracy at distant ranges.

delay-tuned neurons switch on transmission gates that connect a position-dependent voltage through a resistor to a common node. Thus, each neuron is connected to an entry in a lookup table, and when more than a single neuron fires, the appropriate entries for the spiking neurons are averaged. A slow leak to ground is implemented through a resistor much larger than those connected to the transmission gates. This large resistor has little impact on the centroid computation, but creates a fixed estimate of (distant) range to which the system will converge if no delay-tuned neurons are active. The output of the centroid computation is lowpass filtered before being passed to the circuits that control the actual vocalization parameters. Lowpass filtering of the signal prevents sudden jumps in the estimate of distance to the first target on trials in which a transient effect results in a missed echo or generates a response to a nonexistent target. This filtering should not affect tracking performance since it filters out frequencies associated with velocities that are unrealistic for most physical targets. Output of the centroid range estimator is shown for multiple target distances in Figure 9.

\section{Repetition rate and vocalization amplitude}

In addition to reporting the range, the sonar system modifies its vocalization parameters to better collect data, given certain assumptions. Echolocation is an active sampling process, in which a sensory system must emit a signal and wait for a return. The further a target is from the system, the longer it must wait for a return. It is generally assumed that bats cannot distinguish between the echoes from different vocalizations. In such a system, it is important to wait for all echoes to return before emitting another vocalization, because it is difficult to distinguish an echo from a distant target on a previous vocalization from an echo due to a weakly reflect- ing close target on the most recent vocalization. If the system waits for long periods between every vocalization, the rate at which it receives information about its environment decreases.

A possible strategy for overcoming this problem is to select a target or set of targets of interest, and to modify the parameters of the echolocation system in order to obtain as much information as possible about those targets. In order to maximize the rate at which information about the target is received, the system should vocalize at higher rates for close targets where the waiting time for returning echoes is low. At the same time, the system must reduce interference from distant targets due to previous vocalizations. The intensity of a returning echo depends on the reflective properties of the target and on the distance to the target. For a target with fixed reflective properties, the intensity of returning echoes scales approximately with distance $1 / d^{4}$, where $d$ is the distance to the relevant target. By scaling the amplitude of the outgoing vocalization as $d^{4}$, the system can compensate for range-based intensity changes such that the amplitude of the attended echoes remains fixed at a level which will provide sufficient information about the target while reducing the overall amplitude of potentially interfering signals from more distant targets.

The vocalization parameter board alters the vocalization rate and amplitude of the system based on a voltage input which increases with increasing target proximity. Both the increase in repetition rate and the decrease in vocalization amplitude are linearly related to the proximity voltage. Plots of these relationships are shown in Figure 10. In the context of the echolocation system, the vocalization board receives its input from the centroid board. In principle, it is possible to implement nonlinear functions of range such as $d^{4}$, by creating an appropriate lookup table on the centroid board based on the combined functions of $d^{4}$, the ultrasonic transducer response, and the ranges at which the neurons respond. Such a function is not currently implemented. Figure 10 shows plots of vocalization amplitude and rate versus proximity voltage, and Figure 11 demonstrates the system's response to an approaching target.

\section{DISCUSSION}

Neuromorphic VLSI design strives to capture the essential element of any specific instance of neural computation and produce a circuit that will not only reproduce behavior in normal ecological conditions but will also react in qualitatively similar ways to damage and extreme stimulus conditions. The primary purpose is to test these neural algorithms in closed-loop, real-world conditions.

In this paper, we present the design of an analog VLSI circuit that mimics the behavior of delay-tuned neurons in the bat midbrain. The circuits produce the delay-tuned response by implementing the PIR model as supported by numerous neurophysiological and anatomical studies. A population of these neurons is tuned with varying inhibitory durations to produce a range of $\mathrm{BD}$ values. We have incorporated this chip into an artificial bat echolocation system to test these 


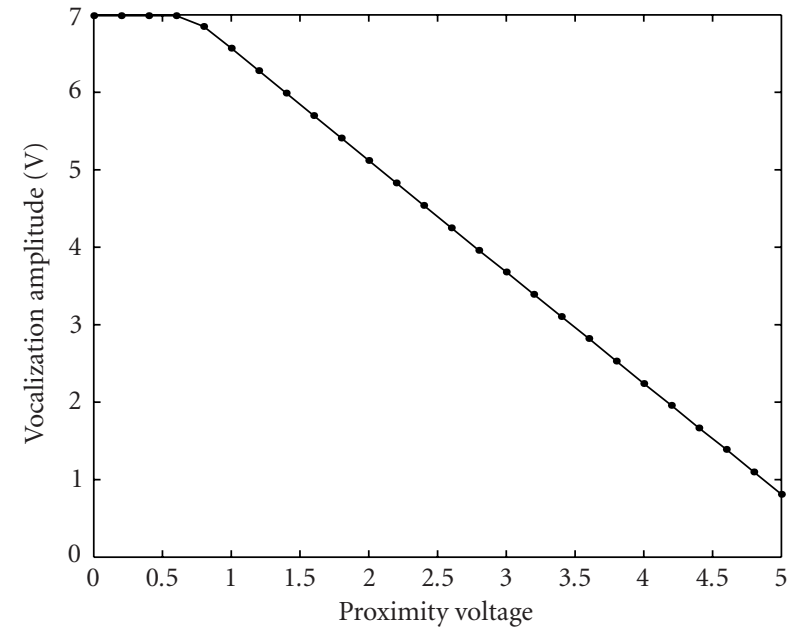

(a)

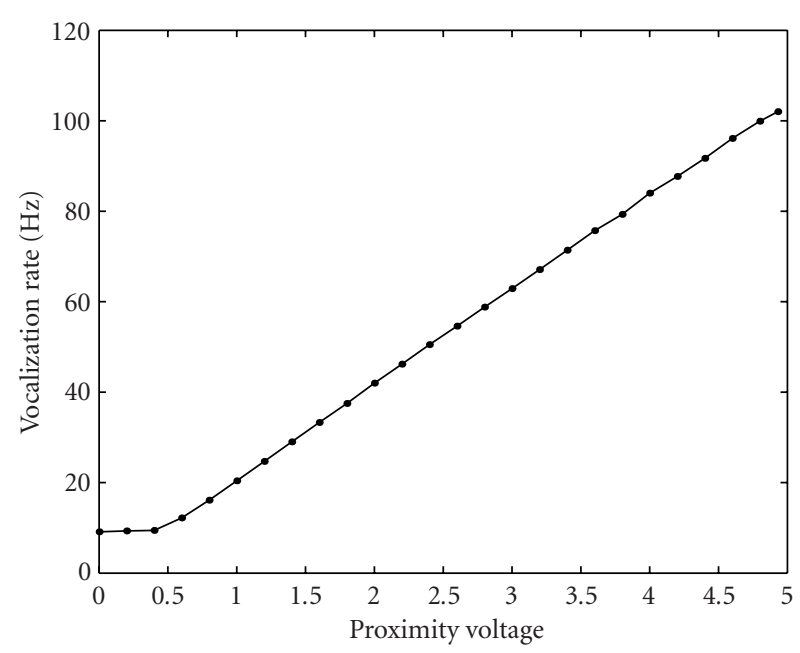

(b)

FIGURE 10: (a) Voltage amplitude of the vocalization signal versus proximity voltage output of the centroid circuit. If the proximity voltage is linear with range, voltage amplitude will be linear with range. (b) Rate of vocalization versus proximity voltage. The vocalization rate must be low enough for any given range so that an echo from that range has time to return before the next vocalization occurs.

neurons in the closed-loop behavior of reporting target range and modifying parameters in response to a moving target.

Our circuit model of the mechanism for PIR is interesting in that it implements dynamic membrane properties, which is not a common tool for neural modelers. The membrane properties of the neuron create a facilitation window that is not defined by the membrane voltage alone but includes the dynamic input impedance of the cell. Furthermore, as a circuit, it implements these membrane dynamics using a simple design that can be used in large arrays of neurons without creating unmanageable biasing problems. The simplified dynamics, however, requires the use of a somewhat unreal-
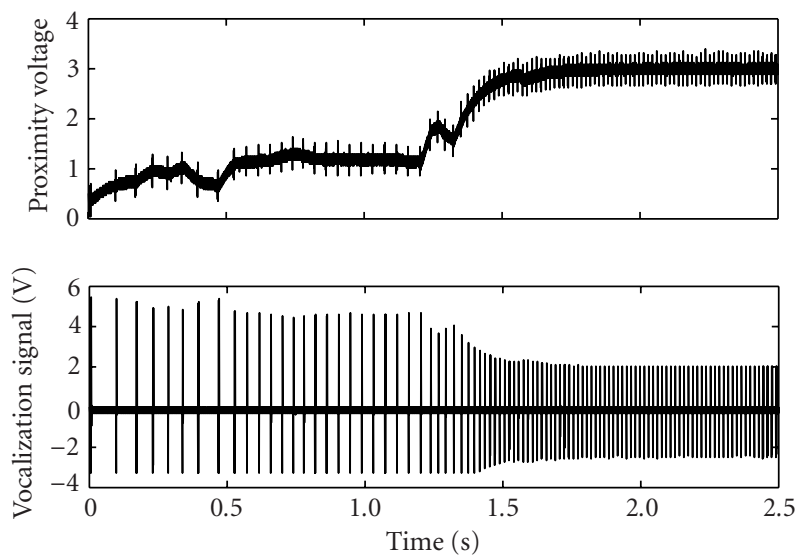

FIGURE 11: Changes in amplitude and repetition rate seen in the system response to an approaching target. Top trace is the proximity voltage from the centroid board. Bottom trace shows the vocalization amplitude and rate as the target approaches.

istically strong inhibition. The chip is capable of measuring target range, and producing an output that can be used to modify the parameters of vocalization. Future implementations of the model will not require a separate threshold circuit, and can use smaller capacitors than those which were used in this version, greatly reducing the size of the neurons. In addition, many of the functions that have been implemented off-chip can be moved into silicon where it will be simpler to implement a more appropriate mapping between range and vocalization amplitude.

This silicon implementation of the delay-tuned neurons of the bat provides a biologically realistic input layer for more detailed neural processing of target range such as attentional tracking and feature binding. While only a small piece of the sophisticated bat echolocation system, these circuits are a critical gateway for processing of range-related information.

Ultimately, our goal is to demonstrate the use of this ranging circuit and others in the aerial flight control of a batsized flying vehicle, an ideal application for miniature, lowpower, real-time, neuromorphic sensory processing circuits.

\section{ACKNOWLEDGMENTS}

The second author is also a member of the Neuroscience and Cognitive Science Program at the University of Maryland. The authors would like to acknowledge the help and advice of Cynthia Moss throughout this project, as well as Shihab Shamma and P. S. Krishnaprasad for their discussions about the basic philosophy and design of auditory computation, Chris Diorio (University Washington) and his group for their assistance with the chip padframes and fabrication, and Adedeji Akinyemi for his assistance in designing the vocalization amplitude control circuit. This work was supported by a DARPA Air-Coupled Microsensors Grant N0001400C0315 and an AFOSR Cooperative Control Grant F496200110415. 


\section{REFERENCES}

[1] M. B. Fenton, "Natural history and biosonar signals," in Hearing by Bats, A. N. Popper and R. R. Fay, Eds., pp. 37-86, Springer-Verlag, New York, NY, USA, 1995.

[2] D. H. Mittmann and J. J. Wenstrup, "Combination-sensitive neurons in the inferior colliculus," Hearing Research, vol. 90, no. 1-2, pp. 185-191, 1995.

[3] J. F. Olsen and N. Suga, "Combination-sensitive neurons in the medial geniculate body of the mustached bat: encoding of target range information," Journal of Neurophysiology, vol. 65, no. 6, pp. 1275-1296, 1991.

[4] A. S. Feng, J. A. Simmons, and S. A. Kick, "Echo detection and target-ranging neurons in the auditory system of the bat Eptesicus fuscus," Science, vol. 202, no. 4368, pp. 645-648, 1978.

[5] H. Riquimaroux, S. J. Gaioni, and N. Suga, "Cortical computational maps control auditory perception," Science, vol. 251, no. 4993, pp. 565-568, 1991.

[6] K. K. Ohlemiller, J. S. Kanwal, and N. Suga, "Facilitative responses to species-specific calls in cortical FM-FM neurons of the mustached bat," Neuroreport, vol. 7, no. 11, pp. 17491755, 1996.

[7] K. H. Esser, C. J. Condon, N. Suga, and J. S. Kanwal, "Syntax processing by auditory cortical neurons in the FM-FM area of the mustached bat Pteronotus parnellii," Proc. Natl. Acad. Sci. USA, vol. 94, no. 25, pp. 14019-14024, 1997.

[8] I. Saitoh and N. Suga, "Long delay lines for ranging are created by inhibition in the inferior colliculus of the mustached bat," Journal of Neurophysiology, vol. 74, no. 1, pp. 1-11, 1995.

[9] T. Hattori and N. Suga, "The inferior colliculus of the mustached bat has the frequency-vs-latency coordinates," Journal of Comparative Physiology A, vol. 180, no. 3, pp. 271-284, 1997.

[10] J. Yan and N. Suga, "The midbrain creates and the thalamus sharpens echo-delay tuning for the cortical representation of target-distance information in the mustached bat," Hearing Research, vol. 93, no. 1-2, pp. 102-110, 1996.

[11] J. J. Wenstrup, D. H. Mittmann, and C. D. Grose, "Inputs to combination-sensitive neurons of the inferior colliculus," Journal of Comparative Neurology, vol. 409, no. 4, pp. 509528, 1999.

[12] C. V. Portfors and J. J. Wenstrup, "Delay-tuned neurons in the inferior colliculus of the mustached bat: implications for analyses of target distance," Journal of Neurophysiology, vol. 82, no. 3, pp. 1326-1338, 1999.

[13] C. V. Portfors and J. J. Wenstrup, “Topographical distribution of delay-tuned responses in the mustached bat inferior colliculus," Hearing Research, vol. 151, no. 1-2, pp. 95-105, 2001.

[14] J. J. Wenstrup and S. A. Leroy, "Spectral integration in the inferior colliculus: role of glycinergic inhibition in response facilitation," J. Neurosci., vol. 21, no. RC124, pp. 1-6, 2001.

[15] W. E. O'Neill and N. Suga, "Encoding of target range and its representation in the auditory cortex of the mustached bat," $J$. Neurosci., vol. 2, no. 1, pp. 17-31, 1982.

[16] S. P. Dear and N. Suga, "Delay-tuned neurons in the midbrain of the big brown bat," Journal of Neurophysiology, vol. 73, no. 3, pp. 1084-1100, 1995.

[17] E. Covey and J. H. Casseday, "The monaural nuclei of the lateral lemniscus in an echolocating bat: parallel pathways for analyzing temporal features of sound," J. Neurosci., vol. 11, no. 11, pp. 3456-3470, 1991.

[18] E. Covey, M. Vater, and J. H. Casseday, "Binaural properties of single units in the superior olivary complex of the mustached bat," Journal of Neurophysiology, vol. 66, no. 3, pp. 1080-1094, 1991.

[19] W. E. Sullivan 3rd, "Possible neural mechanisms of target distance coding in auditory system of the echolocating bat $M y$ otis lucifugus," Journal of Neurophysiology, vol. 48, no. 4, pp. 1033-1047, 1982.

[20] J. A. Winer, D. T. Larue, and G. D. Pollak, "GABA and glycine in the central auditory system of the mustached bat: structural substrates for inhibitory neuronal organization," Journal of Comparative Neurology, vol. 355, pp. 317-353, 1995.

[21] M. Vater, E. Covey, and J. H. Casseday, "The columnar region of the ventral nucleus of the lateral lemniscus in the big brown bat (Eptesicus fuscus): synaptic arrangements and structural correlates of feedforward inhibitory function," Cell Tissue Res., vol. 289, no. 2, pp. 223-233, 1997.

[22] C. Mead, Analog VLSI and Neural Systems, Addison-Wesley, Reading, Mass, USA, 1989.

[23] T. Horiuchi and K. Hynna, "Spike-based VLSI modeling of the ILD system in the echolocating bat," Neural Networks, vol. 14 , no. 67 , pp. 755-762, 2001.

Matthew Cheely received his B.S. degree in marine biology (2000) from the University of North Carolina at Wilmington. He minored in mathematics and did undergraduate research on crustacean neurophysiology. He is currently a Ph.D. student in the Neuroscience and Cognitive Sciences program at the University of Maryland, College Park. His research interests are in computational neuroscience, with emphasis on large-scale spiking neural systems and their implementation in mixed-signal VLSI. He is currently working on projects modeling sensory processing in bat echolocation and the rat whisker system.

Timothy Horiuchi received both his B.S. degree in electrical engineering (1989) and Ph.D. degree in computation and neural systems (1997) from the California Institute of Technology. He did postdoctoral work in the Zanvyl Krieger Mind/Brain Institute at the Johns Hopkins University and is now an Assistant Professor with a joint appointment in the Electrical and Computer Engineering Department and the Institute for

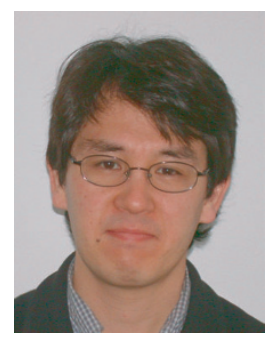
Systems Research at the University of Maryland, College Park (UMCP). He is also a member of the Neurosciences and Cognitive Sciences Program at UMCP. His research interests are in computational neuroscience and the implementation of neural circuit architectures in mixed-mode neuromorphic VLSI-based processors. $\mathrm{He}$ is currently pursuing the development of analog VLSI chips that mimic the signal processing and sensorimotor control of bat echolocation. He is also involved in efforts to improve the tools and techniques used in neurophysiology. 